\title{
Oscillations of magnetic stars: I. Axisymmetric shear Alfvén modes of a spherical shell in a dipolar magnetic field
}

\author{
F. Rincon ${ }^{1,2}$ and M. Rieutord ${ }^{1,3}$ \\ ${ }^{1}$ Laboratoire d'Astrophysique de Toulouse, Observatoire Midi-Pyrénées, 14 avenue É. Belin, 31400 Toulouse, France \\ 2 École Normale Supérieure de Lyon, 46 allée d'Italie, 69364 Lyon Cedex 07, France \\ 3 Institut Universitaire de France
}

Received 15 May 2002 / Accepted 5 November 2002

\begin{abstract}
We carry out an investigation of axisymmetric shear Alfvén waves in a spherical layer of an incompressible resistive fluid when a strong dipolar magnetic field is applied. A decomposition on the spherical harmonics base is used to compute the eigenmodes of the system. Numerical results show that the least-damped Alfvénic modes naturally concentrate near the magnetic polar axis. These modes also show internal shear/magnetic layers associated with resonant field lines. This model is useful when modelling planetary cores sustaining a dynamo, magnetic neutron stars or to the magnetic layer of roAp stars. In this latter case, it shows that shear Alfvén waves provide a good instance of non-perturbative effects due to the strong magnetic field of such stars.
\end{abstract}

Key words. MHD - stars: oscillations - stars: magnetic fields - stars: chemically peculiar

\section{Introduction}

Asteroseismological observations of roAp stars over the last twenty years (Kurtz 1990) have raised the question of the understanding of the oscillations in a star when a global and permanent magnetic field is present. Non-radial oscillations of roAp stars have periods between 4 and 16 min. The observed photometric variations indicate that they are likely high-order, low-degree $p$-modes, perturbed by some physical process related to the presence of a permanent magnetic field, which is known to be mainly dipolar at the surface of these stars. One of the arguments in favor of this interpretation is that the oscillations can be interpreted with the so-called "oblique rotator" model of Kurtz (1990) which considers the oscillations as essentially $\ell=1$ modes aligned with the dipolar magnetic field of the star. Recently, Bigot \& Dziembowski (2002) noticed that this alignment need not be strict and that the pulsation axis lies somewhere between the rotation and magnetic axis.

Balmforth et al. (2001) suggested that oscillations were likely to be excited near the poles of the star rather than near the equator because in the first case, the magnetic field is almost vertical and inhibits convection (in a frozen field picture), allowing some $k$-mechanism excitation, whereas in the second case, magnetic fields lines are nearly horizontal, permitting turbulent convective motions and thus preventing any

Send offprint requests to: F. Rincon,

e-mail: rincon@ast.obs-mip.fr $\kappa$-mechanism. Although this kind of separated treatment between the magnetic pole and the equator is sufficient to recover some properties of the roAp stars, Balmforth et al. (2001) acknowledge that their model has some drawbacks, mainly their neglect of the direct contribution of the Lorentz force to the momentum equation.

Other previous studies by Shibahashi (1983) and Cox (1984) made a more satisfactory treatment of the magnetic field by taking it into account explicitly in the equations. They discovered that convective overstable magneto-gravity modes could be excited at the poles of the star rather than at the equator and found that the oscillation periods of their model were comparable to those observed in roAp stars. However, as they pointed out, they only made a local analysis which is not sufficient to describe the probable global nature of roAp oscillations.

As Biront et al. (1982), Roberts \& Soward (1983) and Campbell \& Papaloizou (1986) explained, the study of oscillations in presence of a magnetic field is a difficult mathematical problem, since magnetic fields can be treated as small perturbations in the interiors of stars but give a major contribution to the pressure in the atmospheres, where regular perturbative methods consequently break down. Dziembowski \& Goode (1996) and Bigot et al. (2000), following up the work of Roberts \& Soward (1983), calculated the shift of $p$-modes frequencies induced by the presence of a strong dipolar magnetic field in a boundary layer approximation. They found that these shifts 
were significant, supporting the idea that the field plays an important role in the mode selection. However, their predictions do not match the observations.

The inferences that we draw from all these studies are of two types: first, they show that many complex processes may explain the observed anisotropy in the oscillations, but that none of them rules the others out. Second, they emphasize the fact that the geometry of the magnetic field plays an essential role in the excitation mechanism of the oscillations in magnetic stars. These two remarks let us think that a general theory of oscillations in magnetic stars including compressibility, stratification, elemental diffusion and radiative transfer is out of reach at the moment. We believe that simpler systems need to be considered in order to progress in the understanding of these astrophysical objects.

For this purpose, we consider in this paper an outrageously simplified star made of an incompressible conducting fluid filling a spherical shell bathed by a dipolar magnetic field. Thus doing we will be able to concentrate on the properties of the global axisymmetric oscillations of such a system, extending in passing the work of Malkus (1967) or Friedlander (1987, 1989) who studied similar systems but with toroidal fields.

Despite its apparent simplicity, this system has many interesting features. First, it is a rather realistic representation of planetary cores sustaining magnetic fields like the ones of the Earth or of the giant planets. In such object density variations are much less important than in stars and the dipolar field is a main component. Other objects like neutron stars also need models in which a strong dipolar magnetic field is taken into account, although in this case Alfvén waves should be replaced by cyclotron-vortex waves (Mendell 1998).

Considering roAp stars, it is clear that we cannot identify shear (solenoidal) Alfvénic modes with the observed pulsations of such stars, which correspond to photometric fluctuations. However, we expect that such waves will be important in more realistic models of these stars. Indeed, if we consider a thin spherical layer like the atmosphere of the star, bathed by a dipolar magnetic field with $B \sim 1 \mathrm{kG}, \rho \sim 10^{-6} \mathrm{~g} / \mathrm{cm}^{3}$ and a thickness of $1000 \mathrm{~km}$, the period of Alfvén modes is $P \sim 440 \mathrm{~s}$, quite comparable to the actual frequencies of roAp stars.

Our system may rather be thought as a preliminary model of the magnetic layer introduced by Roberts \& Soward (1983) which is essential in previous approaches of the oscillations of these stars (Dziembowski \& Goode 1996; Bigot et al. 2000). In these models, the dynamics inside the layer is strongly simplified so as to provide the interior dynamics with tractable boundary conditions. Our system will therefore shed some light on the properties of such layers.

As will be clear below, axisymmetric modes are split into two families: toroidal and poloidal modes. As a first step we will only consider poloidal modes for they couple more easily with acoustic or gravity modes; we surmise that because of these coupling, they can influence the observable modes of magnetic stars.

In the following, we shall first describe the set of equations verified by the perturbations and the numerical technics used to solve them (Sect. 2). Next, the results obtained for axisymmetric poloidal modes are detailed considering the case of standing
(Sect. 3) and propagating modes (Sect. 4). A discussion concludes the paper.

\section{Magnetohydrodynamic waves in a spherical conducting shell}

\subsection{Model}

Since magnetic stars exhibit a permanent and roughly dipolar surface magnetic field, we consider a star with an infinitely electrically conducting core surrounded by a spherical layer of fluid with aspect ratio $\eta$, electrical conductivity $\sigma_{\mathrm{o}}$ and kinematic viscosity $v$. The core is supposed to be the source of a permanent magnetic dipole covering the whole layer. Note that such a partition of the star is necessary in order to avoid the problem of the definition of the magnetic field in the core of the star, the dipole field being singular at the centre. The density $\rho_{\mathrm{o}}$ of the fluid layer is taken to be constant; we thus eliminate phenomena related to compressibility such as $p$-modes (rapid and slow magnetic waves, when a magnetic field is applied). This assumption also removes effects of stratification. Finally, we neglect rotation effects which are known to split the frequencies of oscillation in roAp stars. Classical MHD approximations are used, that is fluid particles have sizes far greater than the typical Debye length of the plasma and all the motions are assumed to be non-relativistic, so that the electric displacement can be neglected in Ampère's law.

\subsection{Governing equations}

We now turn to the mathematical description of the model. The permanent magnetic field (Mc Tavish 2000) is

$\boldsymbol{B}_{\mathrm{o}}=B_{\mathrm{o}}\left(\frac{\cos \theta}{r^{3}} \boldsymbol{e}_{r}+\frac{\sin \theta}{2 r^{3}} \boldsymbol{e}_{\theta}\right)$

using the spherical coordinate base $\left(\boldsymbol{e}_{r}, \boldsymbol{e}_{\theta}, \boldsymbol{e}_{\varphi}\right)$. This field is irrotationnal, so that equilibrium currents vanish in this case. As it was assumed that the fluid is incompressible,

$\boldsymbol{\nabla} \cdot \boldsymbol{v}=0$

Introducing the Lorentz force $\mu_{\mathrm{o}}{ }^{-1}(\boldsymbol{\nabla} \times \boldsymbol{B}) \times \boldsymbol{B}$, we write the momentum equation for an incompressible fluid as

$\frac{\partial \boldsymbol{v}}{\partial t}+\boldsymbol{v} \cdot \boldsymbol{\nabla} \boldsymbol{v}=-\frac{1}{\rho_{\mathrm{o}}} \boldsymbol{\nabla} P+\frac{1}{\rho_{\mathrm{o}} \mu_{\mathrm{o}}}(\boldsymbol{\nabla} \times \boldsymbol{B}) \times \boldsymbol{B}+v \Delta \boldsymbol{v}$

where $\boldsymbol{v}$ is the velocity field and $\boldsymbol{B}$ is the total magnetic field (dipole field plus perturbations); $\mu_{\mathrm{o}}$ is the magnetical permeability of vacuum.

For a conducting fluid, Ohm's law together with Ampère's law yield

$\frac{\partial \boldsymbol{B}}{\partial t}=\boldsymbol{\nabla} \times(\boldsymbol{v} \times \boldsymbol{B})+v_{\mathrm{m}} \Delta \boldsymbol{B}$

where $v_{\mathrm{m}}=\left(\sigma_{\mathrm{o}} \mu_{\mathrm{o}}\right)^{-1}$ is the magnetic diffusivity. Conservation of the magnetic flux states that

$\boldsymbol{\nabla} \cdot \boldsymbol{B}=0$ 
Six boundary conditions are required. On the inner boundary $r=\eta R(\eta<1)$, the magnetic field perturbations have only tangent components because the core is assumed to be infinitely conducting. On the surface, the magnetic field matches the external field which is potential, as there are no currents in the external vacuum. Note that on both boundaries surface currents can occur. As for the velocity field, we choose stress-free conditions (e.g. Chandrasekhar 1961, Chap. 6) on both inner and outer boundaries of the spherical shell

$v_{r}(r=\eta R, \theta)=v_{r}(r=R, \theta)=0$

$\frac{\partial^{2}\left(r v_{r}\right)}{\partial r^{2}}(r=\eta R, \theta)=\frac{\partial^{2}\left(r v_{r}\right)}{\partial r^{2}}(r=R, \theta)=0$.

A non-dimensional form of the previous equations can be obtained by defining the parameters

$V_{\mathrm{A}}=\frac{B_{\mathrm{o}}}{\sqrt{\rho_{\mathrm{o}} \mu_{\mathrm{o}}}}, \quad E=\frac{v}{R V_{\mathrm{A}}}, \quad E_{\mathrm{m}}=\frac{v_{\mathrm{m}}}{R V_{\mathrm{A}}}$

where $V_{\mathrm{A}}$ is the Alfvén speed, $E$ is the inverse of a kinetic Reynolds number and $E_{\mathrm{m}}$ the inverse of the magnetic Reynolds number; for simplicity we will refer to these numbers simply as "diffusivity numbers". We note that $1 / \sqrt{E E_{\mathrm{m}}}$ is just the Hartmann number. In roAp stars, typical values for the various physical quantities are

$B_{\mathrm{o}} \sim 1 \mathrm{kG}, \rho_{\mathrm{o}} \sim 2.5 \mathrm{~kg} \mathrm{~m}^{-3}, R \sim 1.5 R_{\odot}$,

$\sigma_{\mathrm{o}} \sim 6.5 \times 10^{-4} \mathrm{~T}^{3 / 2} \mathrm{ohm}^{-1} \mathrm{~m}^{-1}, T \sim 10^{5} \mathrm{~K}$

thus $V_{\mathrm{A}} \sim 60 \mathrm{~m} \mathrm{~s}^{-1}, E_{\mathrm{m}} \sim 10^{-8}, E \sim 10^{-13}$.

We assume that the background mean flow is zero and study infinitesimal perturbations of the equilibrium state. A time dependence of the form $\mathrm{e}^{\lambda t}$ is assumed, where $\lambda=i \omega+\tau(\tau$ is the damping rate of the solution, $\omega$ is the frequency and $i^{2}=-1$ ), and non-dimensional variables are used:

$$
\begin{aligned}
\boldsymbol{r} & \rightarrow R \boldsymbol{r} \\
\boldsymbol{v} & \rightarrow V_{\mathrm{A}} \boldsymbol{v}(\boldsymbol{r}) \mathrm{e}^{\lambda \mathrm{t}} \\
\boldsymbol{B} & \rightarrow B_{\mathrm{o}} \boldsymbol{B}(\boldsymbol{r})+B_{\mathrm{o}} \boldsymbol{b}(\boldsymbol{r}) \mathrm{e}^{\lambda \mathrm{t}} \\
\rho & \rightarrow \rho_{\mathrm{o}} \\
P & \rightarrow P_{\mathrm{o}}(\boldsymbol{r})+P_{\mathrm{o}} p(\boldsymbol{r}) \mathrm{e}^{\lambda \mathrm{t}}
\end{aligned}
$$

where $\boldsymbol{b}(\boldsymbol{r}), \boldsymbol{v}(\boldsymbol{r})$ and $p(\boldsymbol{r})$ are now first order adimensional quantities. Linearization of Eq. (2)-(5) results in the following MHD set of differential equations

$$
\left.\begin{array}{ll}
\lambda \boldsymbol{\nabla} \times \boldsymbol{v} & =\boldsymbol{\nabla} \times((\boldsymbol{\nabla} \times \boldsymbol{b}) \times \boldsymbol{B})+E \boldsymbol{\nabla} \times \Delta \boldsymbol{v} \\
\lambda \boldsymbol{b} & =\boldsymbol{\nabla} \times(\boldsymbol{v} \times \boldsymbol{B})+E_{\mathrm{m}} \Delta \boldsymbol{b} \\
\boldsymbol{\nabla} \cdot \boldsymbol{v} & =0 \\
\boldsymbol{\nabla} \cdot \boldsymbol{b} & =0
\end{array}\right\}
$$

where the curl of the momentum equation has been taken in order to eliminate the pressure term, and the property $\boldsymbol{\nabla} \times \boldsymbol{B}=0$ has also been used.

\subsection{Local analysis}

For later use, we first recall the dispersion relation of Alfvén waves in a uniform field (Chandrasekhar 1961). From Eqs. (2) and (5), we find that

$-\left(\lambda-v_{\mathrm{m}} k^{2}\right)\left(\lambda-v k^{2}\right)=k^{2} V_{\mathrm{A}}^{2} \cos ^{2} \theta$

where $\theta$ is the angle between the wave vector $\boldsymbol{k}$ and the permanent magnetic field $\boldsymbol{B}$. When there is no ohmic and viscous dissipation, Eq. (11) reduces to

$\frac{\omega}{k}=V_{\mathrm{A}} \cos \theta$.

On the other hand, when non-adiabaticity is taken into account, the damping rate is related to the diffusivity numbers in the following way:

$\tau=-\frac{k^{2}\left(E_{\mathrm{m}}+E\right)}{2}$.

In roAp stars where $E_{\mathrm{m}} \gg E$, the damping of the oscillations is mainly ohmic. In the general case, kinetic and magnetic diffusion play a rather symmetric role.

\subsection{Harmonic projection and symmetries}

To solve the eigenvalue problem expressed by Eq. (10), we project the set of equations on the spherical harmonics in a similar way as in Rieutord $(1987,1991)$. The velocity and magnetic perturbated fields are expanded as follows:

$$
\begin{aligned}
\boldsymbol{v} & \equiv \sum_{\ell=0}^{+\infty} \sum_{m=-\ell}^{+\ell} u_{m}^{\ell} \boldsymbol{R}_{\ell}^{m}+v_{m}^{\ell} \boldsymbol{S}_{\ell}^{m}+w_{m}^{\ell} \boldsymbol{T}_{\ell}^{m} \\
\boldsymbol{b} & \equiv \sum_{\ell=0}^{+\infty} \sum_{m=-\ell}^{+\ell} a_{m}^{\ell} \boldsymbol{R}_{\ell}^{m}+b_{m}^{\ell} \boldsymbol{S}_{\ell}^{m}+c_{m}^{\ell} \boldsymbol{T}_{\ell}^{m} .
\end{aligned}
$$

If $Y_{\ell}^{m}(\theta, \varphi)$ denotes the normalized spherical harmonic, then

$\boldsymbol{R}_{\ell}^{m}=Y_{\ell}^{m} \boldsymbol{e}_{\boldsymbol{r}}, \boldsymbol{S}_{\ell}^{m}=r \boldsymbol{\nabla} Y_{\ell}^{m}$ and $\boldsymbol{T}_{\ell}^{m}=r \boldsymbol{\nabla} \times \boldsymbol{R}_{\ell}^{m}$

where the poloidal parts of the velocity (resp. magnetic) fields $\left(u_{m}^{\ell}, v_{m}^{\ell}\right)\left(\operatorname{resp} .\left(a_{m}^{\ell}, b_{m}^{\ell}\right)\right)$ and its toroidal part $w_{m}^{\ell}\left(\right.$ resp. $\left.c_{m}^{\ell}\right)$ have been introduced. Equation (10) formally reduces to the generalized eigenvalue problem:

$\mathrm{A} x=\lambda \mathrm{B} x$

where $\mathrm{A}$ and $\mathrm{B}$ are differential operators with respect to the $r$ variable only (their expression is given in Appendix A), and

$\boldsymbol{x}_{\lambda m}(r)=\mid \begin{aligned} & \vdots \\ & u_{m}^{\ell}(r) \\ & w_{m}^{\ell}(r) \\ & a_{m}^{\ell}(r) \\ & c_{m}^{\ell}(r) \\ & u_{m}^{\ell+1}(r) \\ & w_{m}^{\ell+1}(r) \\ & a_{m}^{\ell+1}(r) \\ & c_{m}^{\ell+1}(r) \\ & \vdots\end{aligned}$ 
is an eigenvector ( $\ell$ is running from $m$ to $+\infty$ ) associated with the eigenvalue $\lambda$ solution of (16). As an axisymmetric background has been chosen, eigenvectors are given by a single $m$ value (this is a two-dimensional problem). The $v_{m}^{\ell}, b_{m}^{\ell}$ components are omitted because they can be deduced straightforwardly from the others variables by using the incompressibility condition and magnetic flux conservation.

Numerically, we discretize the A and B operators radially on a Gauss-Lobatto grid given by the roots of the Chebyshev polynomial of order $\mathrm{Nr}$, and make a truncation of the problem at a given harmonical degree $\ell=\mathrm{L}$, so that we consider matrices of order $\mathrm{Nr} \times \mathrm{L}$ (see Rieutord \& Valdettaro 1997, for more details). We compute the solutions of the resulting eigenvalue problem with the following tools:

1. a QZ algorithm giving all the eigenvalues $\lambda$ but poor estimations of the eigenvectors;

2. an iterative algorithm for the determination of small subset of modes $\left(\lambda, \boldsymbol{x}_{\lambda m}\right)$ around a given shift in the complex plane.

Couplings between the different components of the fields are introduced by the dipolar field, which breaks the spherical symmetry of the shell. These coupling may be written symbolically

$$
\begin{aligned}
& \mathrm{D}_{1}\left(u_{m}^{\ell}\right)=\mathrm{D}_{2}\left(a_{m}^{\ell+1}\right)+\mathrm{D}_{3}\left(a_{m}^{\ell-1}\right)+i m \mathrm{D}_{4}\left(c_{m}^{\ell}\right) \\
& \mathrm{F}_{1}\left(w_{m}^{\ell}\right)=\mathrm{F}_{2}\left(c_{m}^{\ell+1}\right)+\mathrm{F}_{3}\left(c_{m}^{\ell-1}\right)+i m \mathrm{~F}_{4}\left(a_{m}^{\ell}\right)
\end{aligned}
$$

where $\mathrm{D}_{i}$ and $\mathrm{F}_{i}$ are some discretized differential operators.

In the axisymmetric case $m=0$, eigenvectors look like

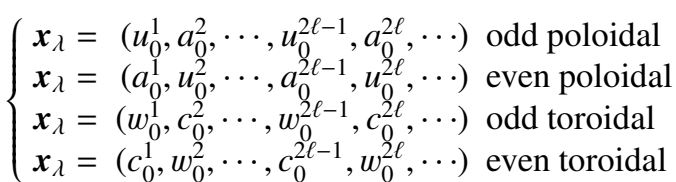

in which even $(+)$ or odd $(-)$ parity specifies whether the velocity field is symmetric or antisymmetric with respect to the equator. Indeed, odd solutions for the magnetic field are even for the velocity field and vice versa. Therefore, each axisymmetric mode has a well defined parity \pm 1 . This is a simple property related to the dipolar background, already emphasized by Dziembowski \& Goode (1996). An other important feature of $m=0$ modes is that the poloidal and toroidal components of both $\boldsymbol{v}$ and $\boldsymbol{b}$ are not coupled.

In the non-axisymmetric case, eigenvectors take the following form:

$$
\boldsymbol{x}_{\lambda m}(r)=\mid \begin{aligned}
& \vdots \\
& u_{m}^{\ell}(r) \\
& w_{m}^{\ell+1}(r) \\
& c_{m}^{\ell}(r) \\
& a_{m}^{\ell+1}(r) \\
& u_{m}^{\ell+2}(r) \\
& w_{m}^{\ell+3}(r) \\
& c_{m}^{\ell+2}(r) \\
& a_{m}^{\ell+3}(r) \\
& \vdots
\end{aligned}
$$

In this latter case it is no longer possible to label the whole mode with a parity \pm 1 . Instead, the toroidal part of the magnetic field has the same parity as the poloidal part of the velocity field and vice versa.

We therefore see that finding a single mode requires the solution of an infinite set of differential equations as a consequence of the coupling introduced by the dipolar field. Of course, the numerical solution needs the system to be truncated at a given harmonic, say L; however, when solutions are converged, the neglected harmonics only represent an exponentially small error compared to the true solution. The resolution needed to achieve convergence depends, as we shall see, on diffusion coefficients which control the size of small-scale structures of the modes (boundary and internal layers). It turns out that the actual stellar values are out of reach numerically; nevertheless, the accessible range of parameters remains sufficiently large to be helpful at deciphering the mode properties. Typically, we could compute modes with fair confidence down to $E \sim E_{\mathrm{m}} \sim 10^{-6}$ using 1700 spherical harmonics and 500 radial grid points.

\subsection{Axisymmetric poloidal modes}

From the foregoing discussion we see that three sub-problems emerge: the one of axisymmetric poloidal perturbations, the one of axisymmetric toroidal perturbations and the one of nonaxisymmetric perturbations. Because of the complexity of their solutions, they need to be considered separately. As already pointed in the introduction, we shall focus here on the first one. This of course does not mean that other types of modes do not play any part. From the numerical point of view, axisymmetric poloidal modes are twice less demanding than their non-axisymmetric counterparts which gives more freedom in the parameter choices.

\section{Diffusion modes}

Poloidal solutions are themselves split into two classes: decaying diffusion modes $(\omega=0, \tau \neq 0)$ and oscillating modes (non-vanishing $\omega$ and $\tau$ ). We first investigate the properties of diffusion modes.

\subsection{Mode structure}

As can be seen in Fig. 1, $\omega=0$ modes are strongly featured by the background magnetic field. In Fig. 2 we compare diffusion modes of opposite parity. We see that symmetric modes for the magnetic field variable $\left(0^{-}\right)$show an empty zone near the equator of the star for both electric current and magnectic field, which is obviously not the case for antisymmetric modes $\left(0^{+}\right)$. It is worth noticing that this empty zone can only be mapped with the internal field lines of the magnetic dipole.

As $\tau$ is necessarily 0 when there is no dissipation, we call these modes diffusion modes because they can only be found when the resistivity does not vanish. Furthermore, a numerical investigation (Fig. 3) shows that the damping rate of a given mode (of a given spatial periodicity) is proportional to $E_{\mathrm{m}}$; we also find that the velocity field is $O\left(E_{\mathrm{m}}\right)$ with respect to the electric current, and does not depend on $E$. 

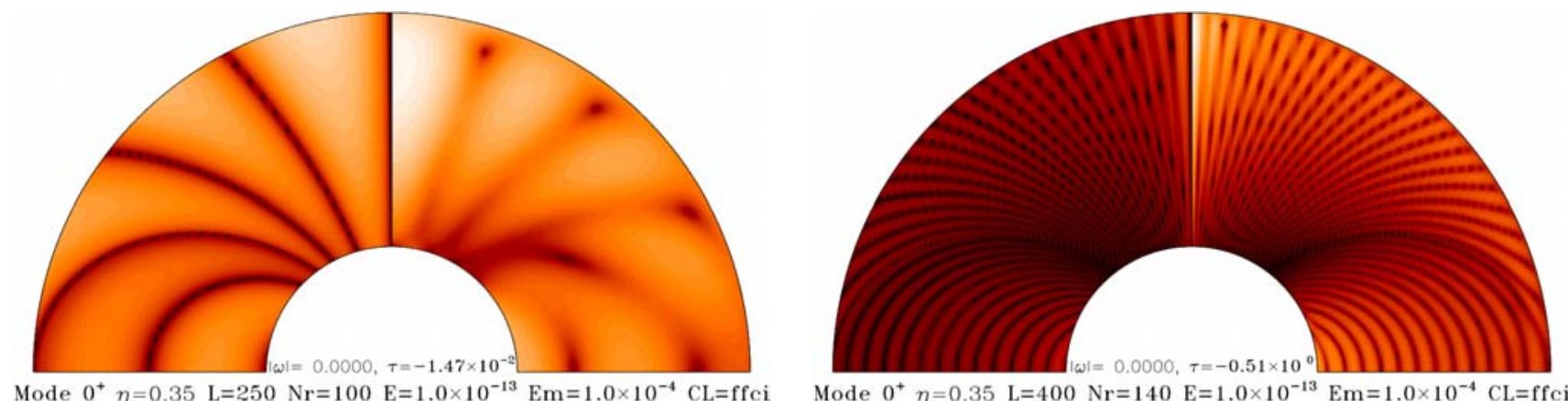

Fig. 1. Meridional section of a shell with aspect ratio $\eta=0.35$, for two stationary odd modes with respect to the magnetic field. For each mode, we plot the magnetic energy $\boldsymbol{b}^{2}$ on a $\log$ color scale on the right quadrant, and the associated ohmic dissipation $(\boldsymbol{\nabla} \times \boldsymbol{b})^{2}$ on the left quadrant. The damping rate obviously increases with the order of the modes. The diffusivity numbers are $E_{\mathrm{m}}=10^{-4}$ and $E=10^{-13}$. The resolution for the radial grid is $\mathrm{Nr}$ points and we used L spherical harmonics.
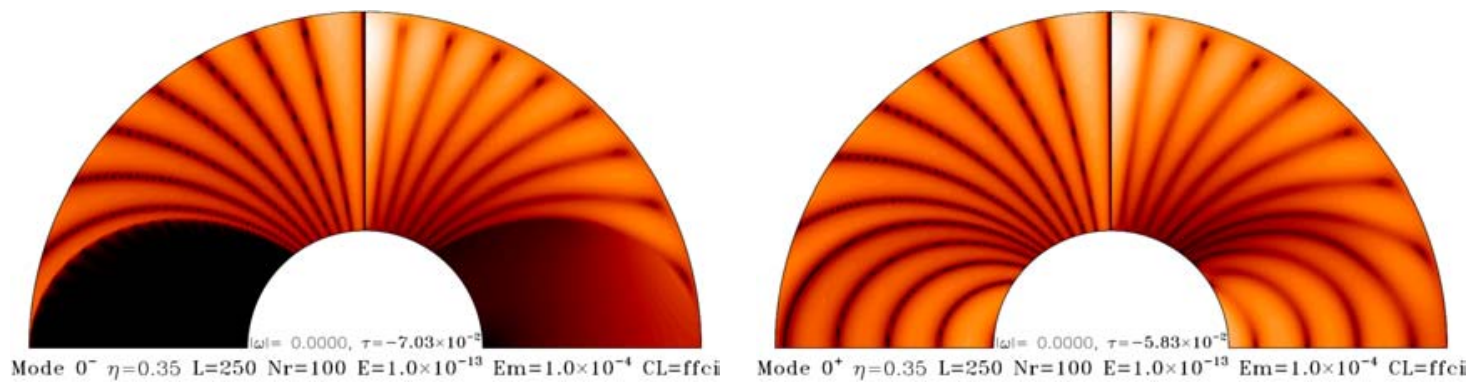

Fig. 2. Comparison between the distribution of magnetic energy and dissipation over the shell for even (left) and odd (right) modes with respect to the magnetic field (same parameters as in Fig. 1). The perturbations vanish for even modes in the equatorial zone, which is mapped by internal magnectic field lines.

\subsection{Interpretation}

As shown by Figs. 1 and 2, the structure of the diffusion modes is closely related to the field lines of the dipole field. This is quite natural if we consider the dispersion relation (12) of Alfvén waves: it states that the pulsation of the oscillations is equal to the Alfvén velocity multiplied by the cosine of the angle between the wave vector and the magnetic field. When $\omega=0$, the wave vector is orthogonal to the field lines. Leaving aside local analysis and using the fact that the observed eigenmodes are highly spatially periodic, we make a global analysis of the equations using WKB theory (see Bender \& Orzag 1978 for an extensive description of WKB methods). Practically, this means that the variations of the permanent magnetic field occur on a far larger length scale than those of the perturbations and therefore we will neglect the former. Using this assumption, Eq. (10) can be rewritten in terms of electric current $\boldsymbol{j}=\boldsymbol{\nabla} \times \boldsymbol{b}$ and vorticity $\boldsymbol{\Omega}=\boldsymbol{\nabla} \times \boldsymbol{v}$ as

$$
\begin{aligned}
& \lambda \boldsymbol{\Omega}=(\boldsymbol{B} \cdot \boldsymbol{\nabla}) \boldsymbol{j}+E \Delta \boldsymbol{\Omega} \\
& \lambda \boldsymbol{j}=(\boldsymbol{B} \cdot \boldsymbol{\nabla}) \boldsymbol{\Omega}+E_{\mathrm{m}} \Delta \boldsymbol{j} .
\end{aligned}
$$

This set of equations is completely symmetric for the magnetic and kinematic variables, which seems to be quite surprising if we look at diffusion modes, for which the velocity field perturbations are small compared to the magnetic field ones. In fact, the difference comes from the boundary conditions met by $\boldsymbol{\Omega}$ and $\boldsymbol{j}$. When the curvature of the spherical coordinates is neglected, stress-free conditions on the velocity field are

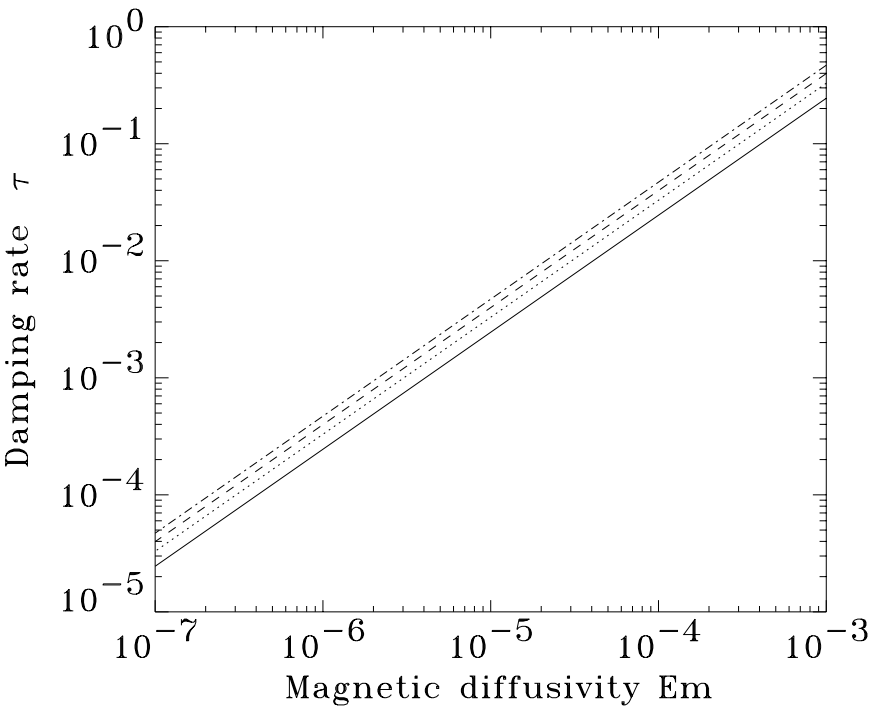

Fig. 3. Evolution of the damping rate of four diffusion eigenmodes with the magnetic diffusivity ( $E$ has been fixed to $10^{-3}$ but $\tau$ does not depend on it for these modes). The aspect ratio is $\eta=0.35$ and a resolution of $L=400$ and $\mathrm{Nr}=100$ has been used.

equivalent to $\boldsymbol{\Omega}=0$ on the boundaries, whereas surface electric currents can occur on the inner and outer spheres. 
Combining equations of system (21) and using $\lambda=\tau$ for diffusion modes, the following relations are obtained in the WKB approximation:

$$
\begin{aligned}
& \tau^{2} \boldsymbol{\Omega}=(\boldsymbol{B} \cdot \boldsymbol{\nabla})^{2} \boldsymbol{\Omega}+\tau\left(E+E_{\mathrm{m}}\right) \Delta \boldsymbol{\Omega}-E E_{\mathrm{m}} \Delta \Delta \boldsymbol{\Omega} \\
& \tau^{2} \boldsymbol{j}=(\boldsymbol{B} \cdot \boldsymbol{\nabla})^{2} \boldsymbol{j}+\tau\left(E+E_{\mathrm{m}}\right) \Delta \boldsymbol{j}-E E_{\mathrm{m}} \Delta \Delta \boldsymbol{j} .
\end{aligned}
$$

We assume that $\boldsymbol{\Omega}, \boldsymbol{j}$, and $\tau$ can be expanded in series involving powers of $E_{\mathrm{m}}$ and $E$ :

$$
\begin{aligned}
& \boldsymbol{\Omega}=\boldsymbol{\Omega}_{\mathrm{o}}+f_{1}\left(E, E_{\mathrm{m}}\right) \boldsymbol{\Omega}_{1}+\cdots+f_{n}\left(E, E_{\mathrm{m}}\right) \boldsymbol{\Omega}_{n} \\
& \boldsymbol{j}=\boldsymbol{j}_{\mathrm{o}}+f_{1}\left(E, E_{\mathrm{m}}\right) \boldsymbol{j}_{1}+\cdots+f_{n}\left(E, E_{\mathrm{m}}\right) \boldsymbol{j}_{n} \\
& \tau=f_{1}\left(E, E_{\mathrm{m}}\right) \tau_{1}+\cdots+f_{n}\left(E, E_{\mathrm{m}}\right) \tau_{n}
\end{aligned}
$$

where the $f_{n}$ are functions of the diffusivity numbers at a given power $n$ only ( $\tau_{\mathrm{o}}=0$ results from the fact that there is no damping in absence of dissipation processes). Using order of magnitudes in powers of $E$ and $E_{\mathrm{m}}$, Eq. (21) result in

$$
\begin{aligned}
& (\boldsymbol{B} \cdot \boldsymbol{\nabla}) \boldsymbol{\Omega}_{\mathrm{o}}=0 \\
& (\boldsymbol{B} \cdot \boldsymbol{\nabla}) \boldsymbol{j}_{\mathrm{o}}=0
\end{aligned}
$$

at zeroth order; hence, zeroth order variables are functions of the field line on which they are calculated only.

We introduce dipolar coordinates $\mu$ and $v$ (Radoski 1967; Cummings \& O'Sullivan 1969) to map a meridional section of the star:

$\mu=\frac{\cos \theta}{r^{2}}, \quad v=\frac{\sin \theta}{\sqrt{r}}$.

Using these coordinates, a new orthogonal set of base vectors can be defined, one with $\mu$ that is tangent to the field lines and one with $v$ orthogonal to the field lines. With these new variables, Eq. (24) states that the field lines indexed by $v$ form a web of characteristics. As boundary conditions on $\boldsymbol{\Omega}$ are $\boldsymbol{\Omega}(r=\eta)=\boldsymbol{\Omega}(r=1)=0$ whatever $\theta$ (and therefore whatever $v), \boldsymbol{\Omega}_{\mathrm{o}}=0$ all over the star, as the boundary conditions propagate along the characteristics. Note that the situation is quite different for the electric current $\boldsymbol{j}_{\mathrm{o}}$, which does not verify such boundary conditions. Therefore $j$ is at least one order larger than $\boldsymbol{\Omega}$ in terms of powers of the diffusivity numbers, as the numerical results show.

The empty zone in Fig. 2 can be understood in the same way. For axisymmetric magnetic poloidal even modes, $j$ is purely toroidal and vanishes in the equatorial plane because of the symmetry condition on $\boldsymbol{b}$. According to Eq. (24) this property propagates along the field lines (which are in this case internal field lines, i.e. field lines that intersect the equatorial plane inside the shell), so that there can be no currents and magnetic field in the whole equatorial zone mapped by internal field lines.

It is difficult to tell more about this kind of modes. Despite their apparent simplicity, no analytic solution could be found. The reason lies, as expected, in the probable absence of any suitable coordinate system which allows the separation of variables. For instance, we observe that in the diffusionless case $\boldsymbol{j}$ obeys

$$
(\boldsymbol{j} \cdot \boldsymbol{\nabla}) \boldsymbol{B}=(\boldsymbol{B} \cdot \boldsymbol{\nabla}) \boldsymbol{j}
$$

The general solution for this equation is

$\boldsymbol{j}=j \boldsymbol{e}_{\varphi}=H(v) r^{3 / 2} \boldsymbol{e}_{\varphi}$

where $H(v)$ is an undetermined function; despite the fact that the dependence of $j$ with $r^{3 / 2}$ is conspicuous numerically, no differential equation can be obtained for $H$ only because $\Delta\left(H(v) r^{3 / 2}\right)$ is not separable.

\section{Oscillatory modes}

We now turn to the most interesting modes, namely oscillatory modes, as they may be related to the observed periodic oscillations of roAp stars.

\subsection{The eigenvalue spectrum}

We first consider the properties of eigenvalues (i.e. eigenfrequencies and damping rates) when the parameters are varied. In Fig. 4 we plot the distribution of eigenvalues in the complex plane for $E=10^{-13}, E_{\mathrm{m}}=10^{-4}, \eta=0.35$. The regular distribution which we observe shows that each eigenvalue is associated with an eigenfunction whose structure is dominated by non-diffusive effects. The regular spacing indeed shows that no small-scale effects control the energy containing features; the opposite situation occurs for instance with inertial modes (modes sustained by Coriolis force in rotating fluids see e.g. Rieutord et al. 2001).

As expected for a two dimensional problem, eigenvalues are determined by two quantum numbers. Quite strikingly, the frequencies seem to depend only on one quantum number. The damping rates vanish when diffusion effects vanish: as shown in Fig. 5, the damping rates are roughly proportional to $\max \left(E, E_{\mathrm{m}}\right)$; actually, the convergence to the adiabatic frequency follows the same law.

The vanishing of the real part of the eigenfrequencies in the adiabatic limit raises the possibility of a singular behaviour of the frequency spectrum in this limit. Indeed, either the eigenvalues of a given horizontal branch all collapse at the same frequency which are thus infinitely degenerate or they densely cover the frequency axis yielding a continuous spectrum; a third possibility is that eigenvalues all disappear if eigenfunctions lose their square integrability. As will be shown below, the existence of internal boundary layers make the adiabatic limit singular but the exact nature of the singularity is still an open question.

A final comment in Fig. 4 concerns the line $\omega(\tau)$ followed by least-damped modes; it is the expression of the dispersion relation of Alfvén waves since the damping rates $\tau$ roughly scales with the square of the wavenumber.

\subsection{Associated eigenfunctions}

The foregoing properties of the eigenvalues may let us think that the eigenmode structure may be computed with a simple adiabatic model, just like acoustic or gravity modes in a spherical star. The situation is in fact more complex but as we 


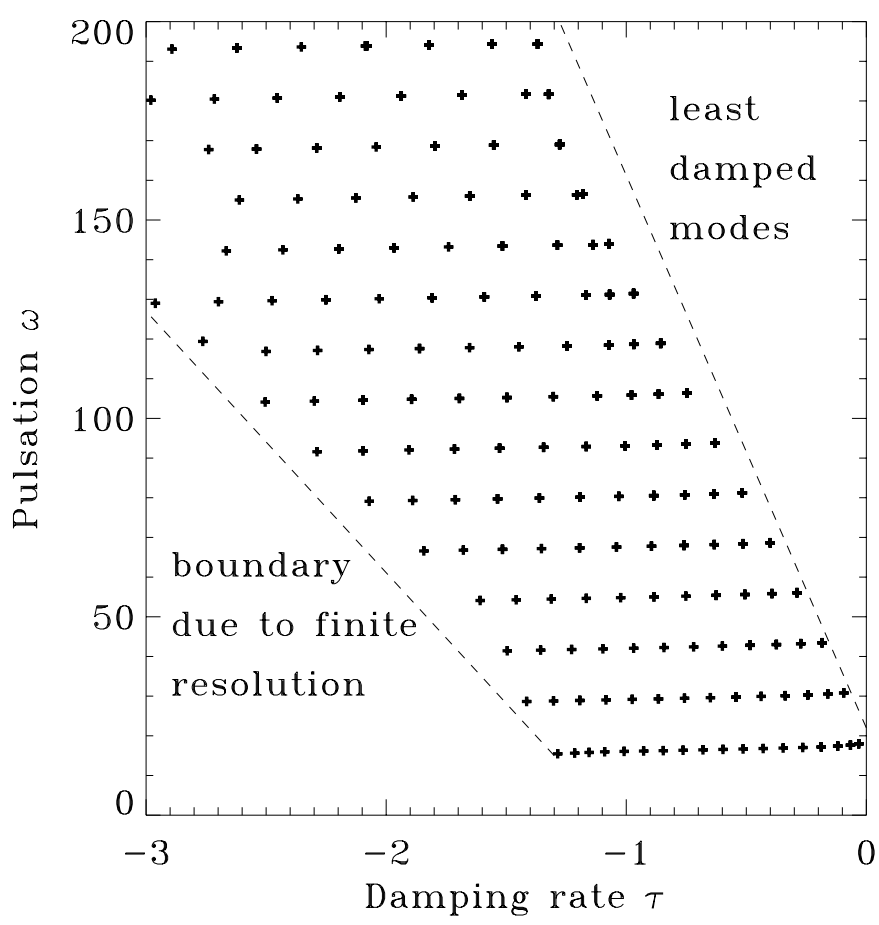

Fig. 4. Spectrum of the eigenvalues in the complex plane for a poloidal axisymmetric configuration with $\eta=0.35, E_{\mathrm{m}}=10^{-4}, E=10^{-13}$. The bottom-left region of the plane is empty because the resolution needed is too large for the calculator, but eigenvalues should be found in this zone. On the contrary, no eigenvalue is found in the top-right region as a consequence of the dispersion relation between the pulsation and the wavenumber (or damping rate).

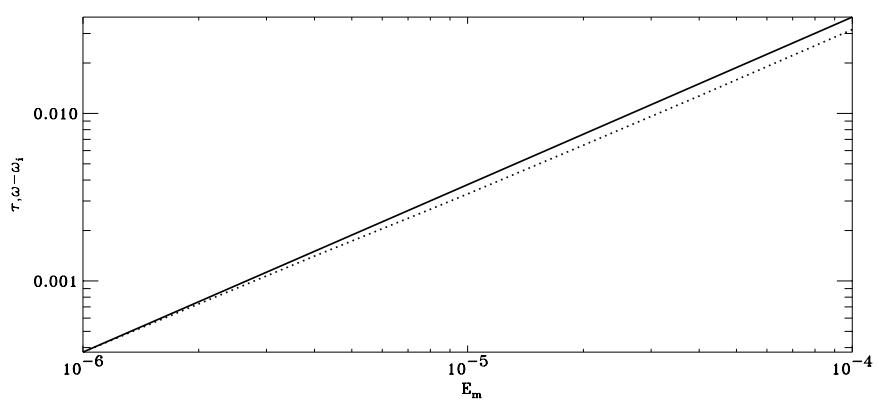

Fig. 5. Dependence of the damping rate and difference between the real and inviscid eigenfrequencies with respect to $E_{\mathrm{m}}$ when $E=10^{-10}$. We see that the damping rate quite closely follows the linear law in this range of values.

shall see modes can be split into their adiabatic part and nonadiabatic complement. We first analyse the adiabatic part which is the main component.

\subsubsection{Adiabatic features}

First, as we noted, eigenfrequencies seem to depend only on one quantum number. A look at the actual eigenmodes explains such a situation. As shown in Fig. 6 modes of similar frequency but different damping rates are characterized by different number of nodes in the direction perpendicular to the field lines.
On the other hand, modes of different frequency possess a different number of nodes in the field lines direction (see Fig. 7). Recalling that in local analysis

$\omega=k_{x} B_{x}+k_{z} B_{z}$

it is obvious that the variation of $\omega$ when $k_{x}$ is incremented ( $k_{z}$ remains constant) is far smaller than the variation of $\omega$ when $k_{z}$ is changed at constant $k_{x}$, because $B_{z} \gg B_{x}$ near the pole where the eigenmodes are found. Therefore the dipolar field is responsible for the existence of Alfvén waves in both directions and the ratio of the intensity of the field in the two directions is responsible for the quasi-degeneracy of the eigenfrequencies with respect to the horizontal wavenumber.

To further illustrate the weak dependence of eigenfunctions on the horizontal (compared to vertical) structure of the modes, we consider the following simplified model describing polar regions: a horizontal perfectly conducting inviscid fluid layer is bathed by a vertical magnetic field whose strength decreases with $1 / z^{3}$ (we neglect the horizontal component of the field); not surprisingly the layer lies between $z=\eta$ and $z=1$. Moreover, we consider the high frequency limit and thus make a WKB analysis. Neglecting diffusivities, we find that velocity and magnetic field perturbations must verify:

$i \omega \boldsymbol{b}=B(z) \frac{\partial \boldsymbol{v}}{\partial z}$

and

$i \omega \boldsymbol{v}=-\nabla p+\boldsymbol{j} \times \boldsymbol{B}$.

We further simplify this system, considering perturbations $\boldsymbol{v}=$ $v(z) \boldsymbol{e}_{x}$ and $\boldsymbol{b}=b(z) \boldsymbol{e}_{x}$, where $\boldsymbol{e}_{x}$ is the unit vector along the $x$ axis. From the curl of Eq. (30) and the use of Eq. (29), we find that

$\frac{\mathrm{d}^{3} v}{\mathrm{~d} z^{3}}+\omega^{2} z^{6} \frac{\mathrm{d} v}{\mathrm{~d} z}=0$

which can be solved at zeroth order (in $1 / \omega$ ) by

$v(z)=A \exp \left(\frac{i \omega z^{4}}{4}\right)+B \exp \left(-\frac{i \omega z^{4}}{4}\right)$

The behaviour of the solution as $\exp \left(i \omega z^{4} / 4\right)$ is not suprising and consistent with the non-regular spacing of the nodes of the eigenfunctions (see Fig. 7). Boundary conditions for $v(z)$ are simply that $v=0$ at $z=\eta$ (no motion of the field lines is authorized as they are frozen in a solid). At $z=1$, which is the interface between the fluid and the vacuum, no boundary condition is required, since the motion is parallel to the boundary, $\boldsymbol{b}$ needs just be continuous. Thus, a whole class of velocity fields may solve the problem. However, we are more particularly interested in those which verify $v^{\prime}(z)=0$ at $z=1$ like in our original problem where stress-free boundary conditions are used. Selecting these velocity fields yields the following quantization of the frequencies:

$\omega_{n}=\frac{2(2 n+1) \pi}{1-\eta^{4}}$ 


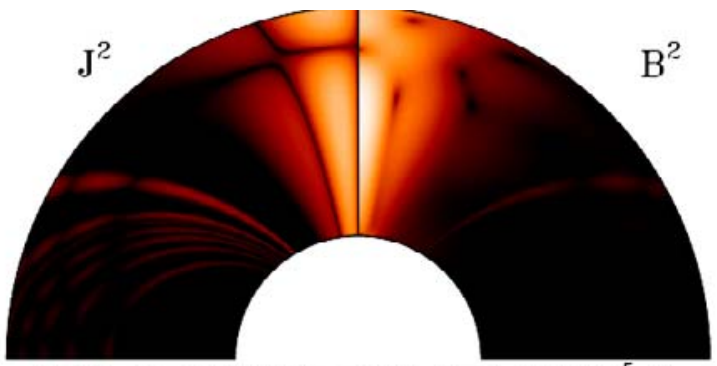

Mode $0^{+} \eta=0.35 \mathrm{~L}=1000 \mathrm{Nr}=300 \mathrm{E}=1.0 \times 10^{-5} \quad \mathrm{E}_{\mathrm{m}}=1.0 \times 10^{-5}$ $|\omega|=17.996 .3 \tau=-5.38 \times 10^{-3}$

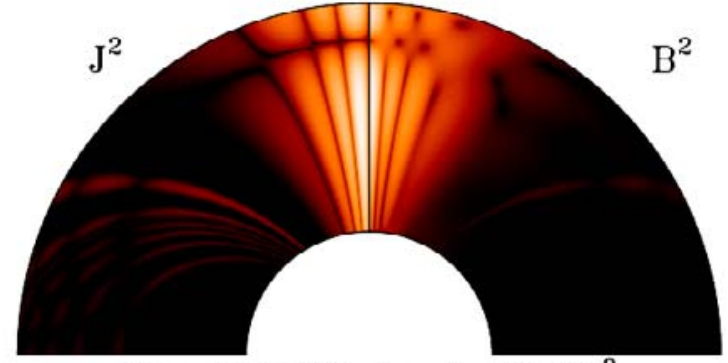

$|\omega|=17.4069 \tau=-2.11 \times 10^{-2}$

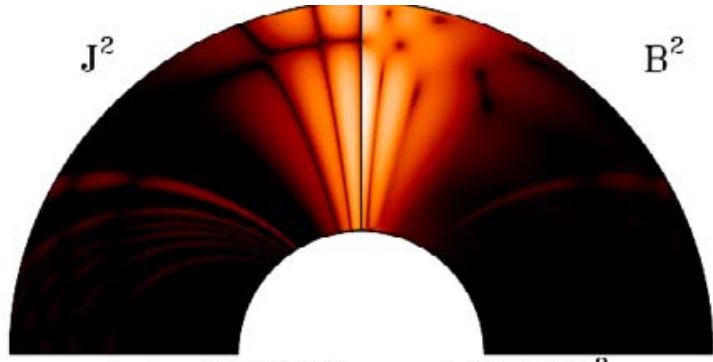

$|\omega|=17.6416 \tau=-1.13 \times 10^{-2}$

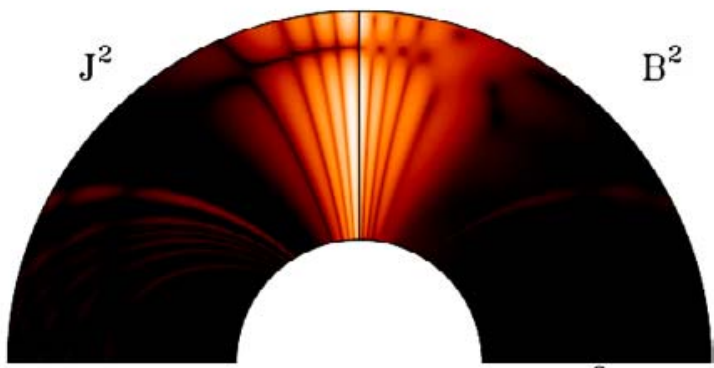

$|\omega|=17.2444 \tau=-3.58 \times 10^{-2}$

Fig. 6. Magnetic dissipation and magnetic energy in a meridional plane for four eigenmodes around frequency 18 . They all share the same number of nodes along the field lines but have different wavenumbers in the orthogonal direction. We used $E_{\mathrm{m}}=10^{-5}, E=10^{-5}$ and $\eta=0.35$, $L=1000$ spherical harmonics and 300 radial grid points. Note that the resonant field lines are slightly visible on the magnetic dissipation.
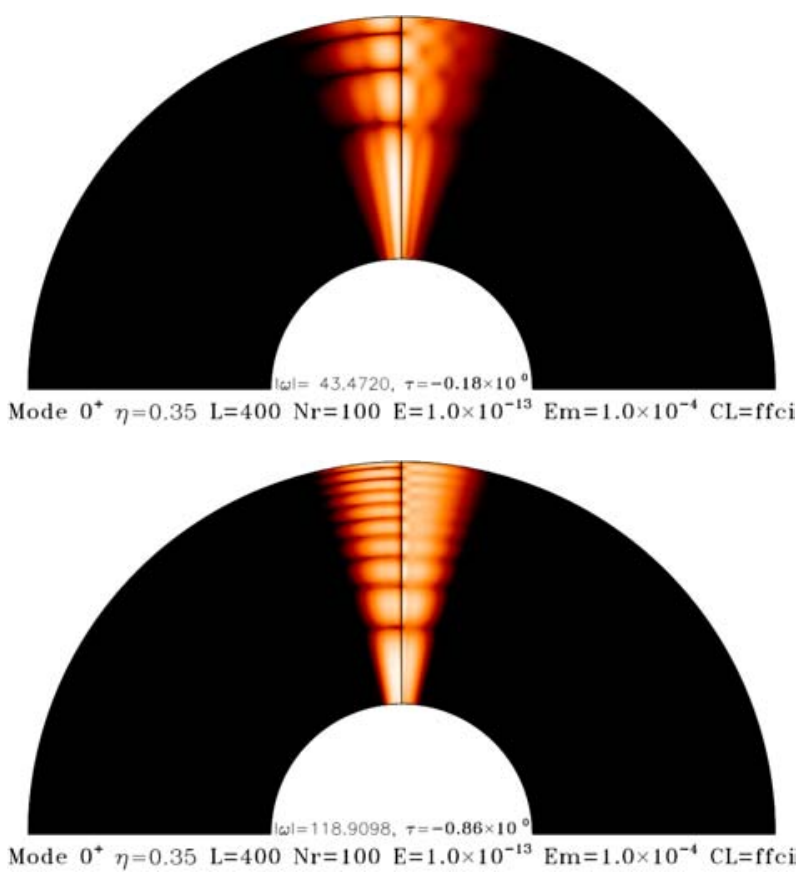
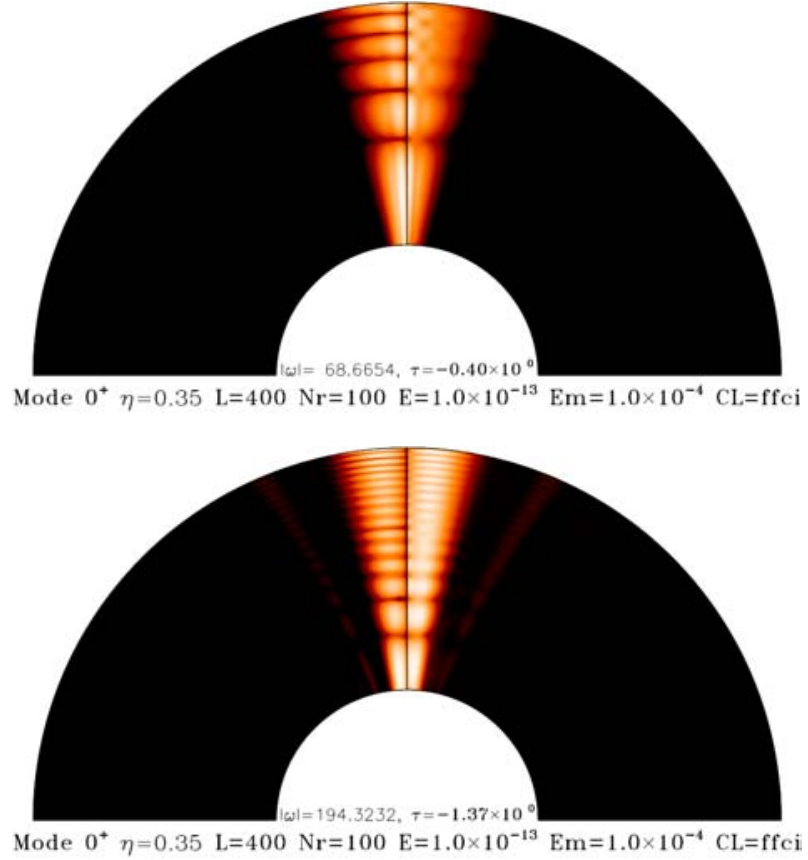

Fig. 7. Meridional distribution of electric current (left) and magnetic energy (right) for four given eigenmodes with $E_{\mathrm{m}}=10^{-4}, E=10^{-13}$ and $\eta=0.35$. Each mode has a different radial periodicity and pulsation. The main feature of these waves is the localization of energy near the dipolar axis.

As shown by Table 1, these frequencies are surprisingly close to those given by numerical solutions. Moreover, the frequency spacing $\Delta \omega=\omega_{n+1}-\omega_{n}=4 \pi /\left(1-\eta^{4}\right)$ is in very good agreement with the numerical results as shown in Fig. 8.

Another striking feature of the axisymmetric Alfvén modes is their localization on the polar caps. As the preceding results show, the dynamics of these modes is mostly adiabatic and therefore this other property is also contained in the dissipationless equations. However, even thus simplified the problem remains cumbersome. We found more illuminating to consider a toy problem where the characteristic features of the background magnetic field are partly simplified. Thus, as above, 
Table 1. Comparison between the simple WKB model and the actual frequencies issued from the resolution of the complete system Eq. (10). the parameters used for the numerical solutions are $\eta=0.35, E=10^{-10}, E_{\mathrm{m}}=10^{-5}$ using 1000 spherical harmonics and 300 radial grid points.

\begin{tabular}{lll}
\hline \hline$n$ & $\omega_{n}$ & $\omega_{\text {num }}$ \\
\hline 1 & 19.14 & 18.00 \\
2 & 31.89 & 30.82 \\
3 & 44.65 & 43.50 \\
4 & 57.41 & 56.14 \\
5 & 70.17 & 68.77 \\
6 & 82.93 & 81.40 \\
7 & 95.68 & 94.02 \\
8 & 108.4 & 106.7 \\
9 & 121.2 & 119.3 \\
\hline
\end{tabular}

we consider a horizontal inviscid and perfectly conducting fluid layer bathed by a vertical magnetic field whose intensity now depends on the horizontal coordinate, i.e. we assume $\boldsymbol{B}=B(x) \boldsymbol{e}_{z}$. Within such a background we examine the necessary conditions for the existence of Alfvén modes.

To mimic poloidal oscillations, we need to take perturbations of the velocity and magnetic fields which are of the form $\boldsymbol{v}=v(x, z) \boldsymbol{e}_{x}, \boldsymbol{b}=b(x, z) \boldsymbol{e}_{x}$. A toroidal field would be along the $y$-axis. As shown in appendix, the perturbations obey

$\frac{\partial^{2} v_{x}}{\partial x^{2}}+2 k_{\|}^{2} \frac{B^{\prime}}{B\left(k_{\|}^{2}-\frac{\omega^{2}}{B^{2}}\right)} \frac{\partial v_{x}}{\partial x}-k_{\|}^{2} v_{x}=0$

where primes denote $x$-derivatives. This equation also shows that a dispersion relation like (12) is no longer valid in a nonuniform magnetic field.

Equation (33) shows that eigenmodes are trapped around a maximum of $B(x)$. Let us indeed consider a magnetic field of the form $B=\exp \left(-x^{2}\right)$. in this case

$\frac{\partial^{2} v_{x}}{\partial x^{2}}-\frac{4 k_{\|}^{2} x}{\left(k_{\|}^{2}-\omega^{2} \exp \left(2 x^{2}\right)\right)} \frac{\partial v_{x}}{\partial x}-k_{\|}^{2} v_{x}=0$.

At $x_{\mathrm{c}}$ such that $B\left(x_{\mathrm{c}}\right)=\omega / k_{\|}$, the equation is singular and solutions should be searched for in the two domains $0 \leq x<x_{\mathrm{c}}$ or $x>x_{\mathrm{c}}$. Symmetry considerations impose that $v_{x}=0$ for $x=0$. In the first domain the solution is not known analytically, however, if $x>x_{\mathrm{c}} \gg 1$, the solution are exponentially decaying, i.e. $v_{x}=\exp \left(-k_{\|} x\right)$, thus showing the trapping of the modes.

We therefore understand that in our spherical system where the field intensity is maximum at the poles $\left(B_{z}^{2} \propto \cos ^{2} \theta\right)$, Alfvén modes are localized on polar caps.

\subsubsection{Non-adiabatic effects}

We already observed one non-adiabatic effect: the removal of the quasi-degeneracy of modes with the same number of nodes along the field lines. We know that such a situation does not occur with other type of modes like gravity or acoustic ones which can be calculated with an adiabatic model. Indeed, these

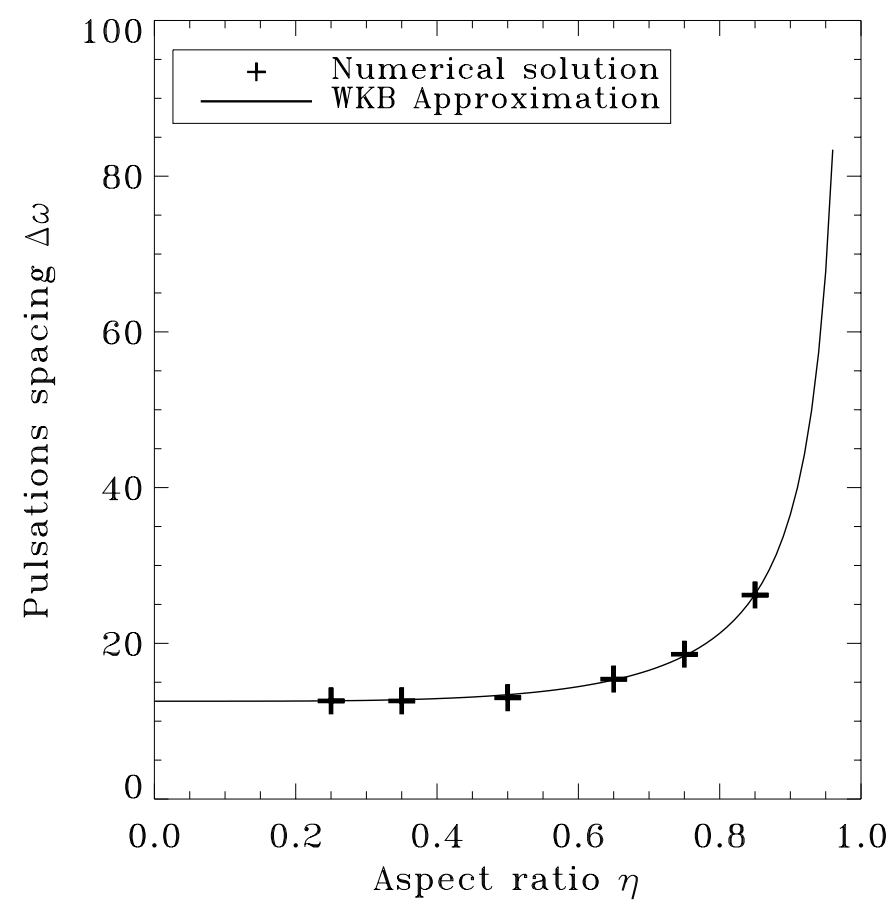

Fig. 8. Spacing of the observed pulsations as a function of the aspect ratio $\eta$. The numerical values are plotted with crosses and are compared to the WKB approximation of the solution (solid line) $\Delta \omega=4 \pi /\left(1-\eta^{4}\right)$.

modes are usually computed in a spherically symmetric background which authorizes the separation of variables. In such a case, the problem is one dimensional for each spherical harmonic component and degeneracy of frequencies $\omega_{n l}$ is accidental.

The introduction of non-adiabatic effects, namely viscosity and magnetic diffusion, solves this problem but also introduces numerical difficulties. These are associated with the appearance of boundary layers and internal shear layers. The formers are the well-known Hartmann layers which are in general extremely thin, i.e. scaling like $\sqrt{E E_{\mathrm{m}}}$; choosing stressfree boundary conditions minimizes their influence on the solutions; other techniques are also available to remove them (Potherat et al. 2002). Internal layers are certainly nastier features and we discovered that they strongly limit the computation of eigenmodes.

Internal layers come into play through resonant field lines. Indeed, if we consider a mode with $n$ nodes along the field lines and located around the pole, then it is easy to see that for the same frequency, other field lines, which are necessarily longer and weaker, will be resonant with $n+1, n+2, \ldots$ nodes, until the critical field line, which connects the inner boundary to the outer shell equator, is reached. This feature of the modes is clearly illustrated in Fig. 9 using a thin spherical shell with $\eta=0.9$. There, the main part of the mode has one node and we can identify resonant lines up to $n=7$. In passing, we see that the thickness of the shell does not change the broad properties of the modes. 


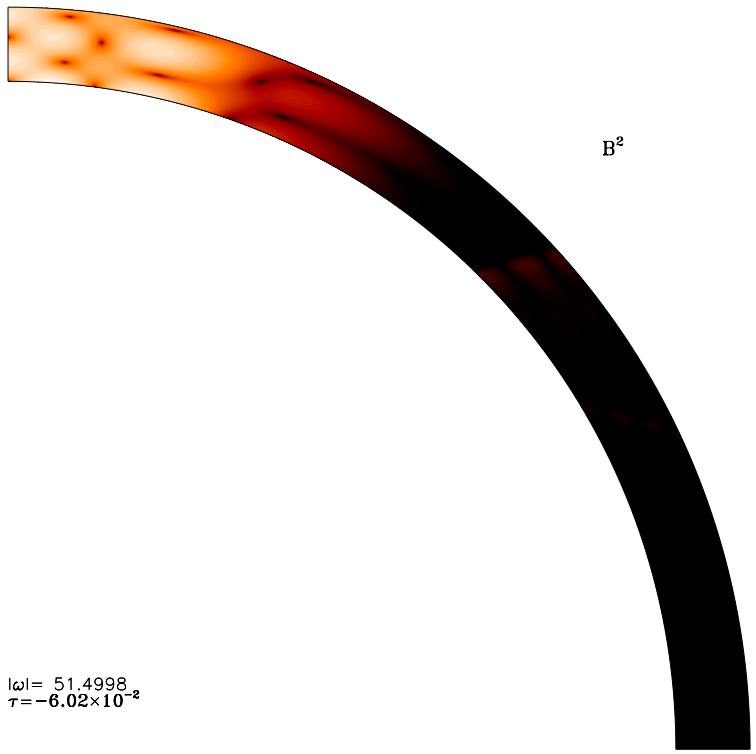

Mode $0^{+} \eta=0.90 \mathrm{~L}=900 \mathrm{Nr}=80 \mathrm{E}=1.0 \times 10^{-6} \mathrm{E}_{\mathrm{m}}=1.0 \times 10^{-8}$

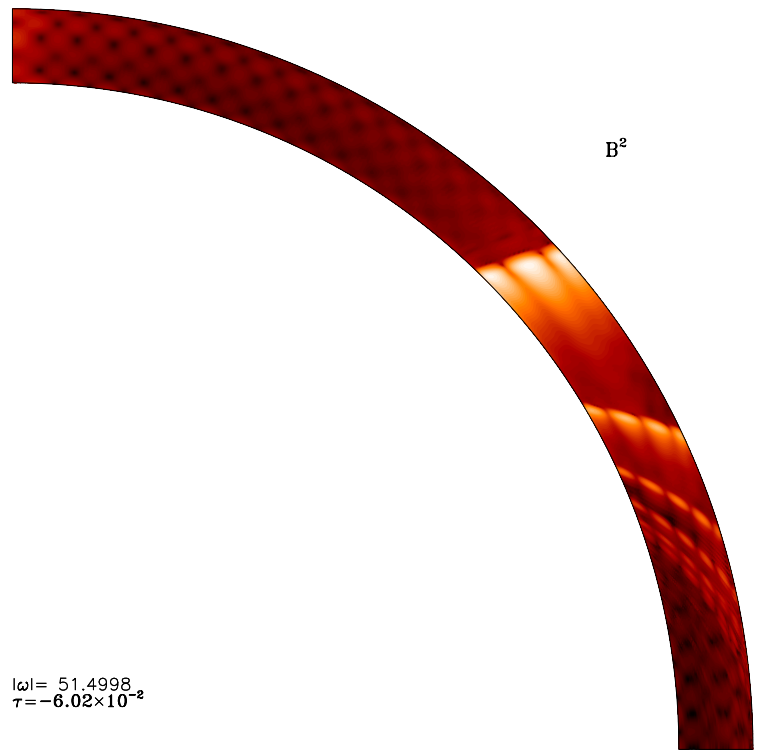

Mode $0^{+} \eta=0.90 \mathrm{~L}=900 \mathrm{Nr}=80 \mathrm{E}=1.0 \times 10^{-6} \mathrm{E}_{\mathrm{m}}=1.0 \times 10^{-6}$

Fig. 9. Left: the magnetic energy of the mode $n=1$ in a thin shell with $\eta=0.9$; like the thick shell case, the amplitude of the mode is located on the polar cap. Right: the adiabatic part of the mode has been filtered out and the resonant field lines which are barely visible on right are now clearly visible from $n=2$ up to $n=7$.
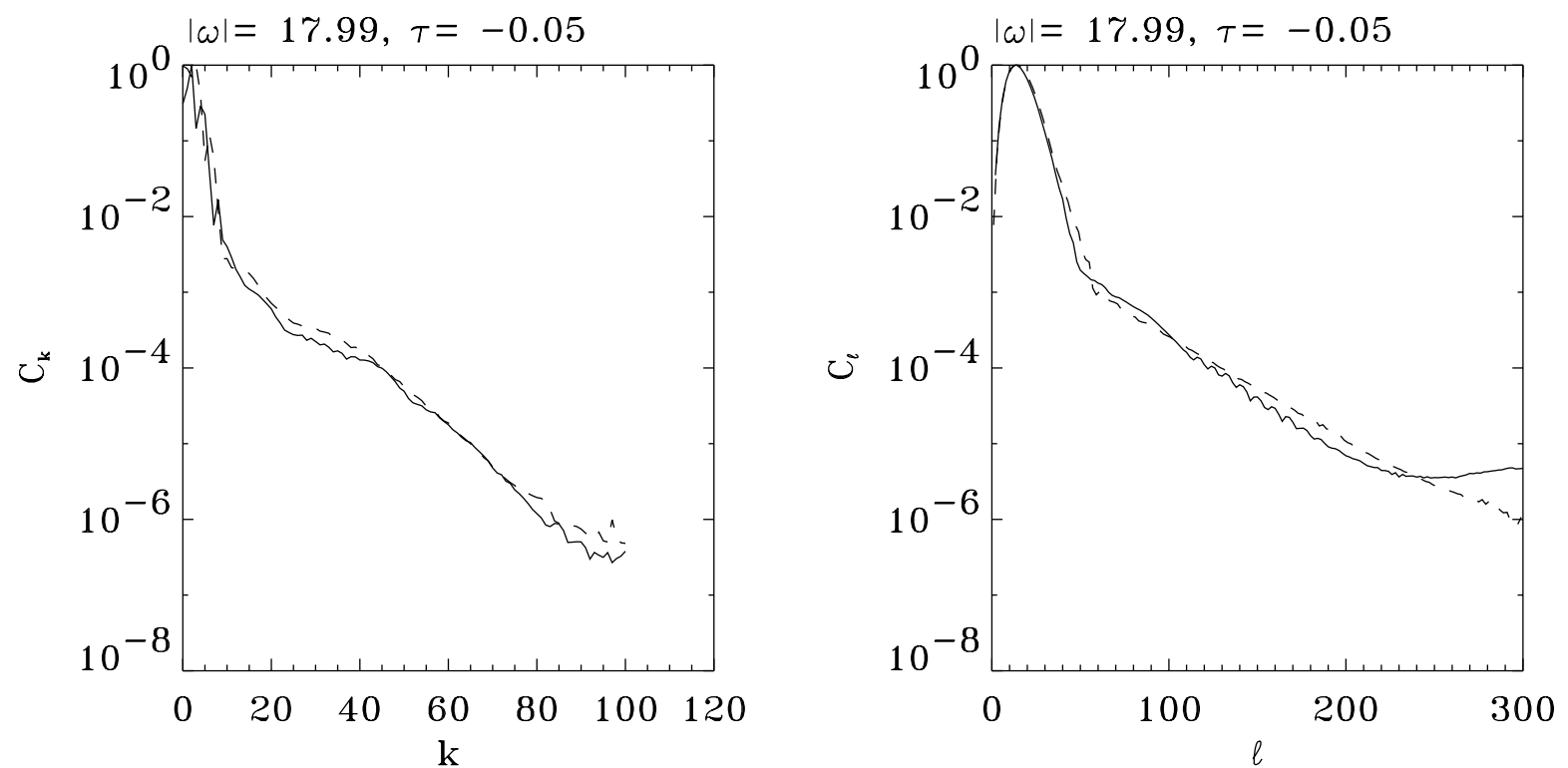

Fig. 10. Spectral decomposition of the magnetic (solid line) and velocity (dashed line) fields for the first mode of Fig. 6 when $E=E_{\mathrm{m}}=10^{-4}$. Left: the maximum, over all spherical harmonic components, of the absolute value of the Chebyshev coefficients. Right: the maximum, over all Chebyshev coefficients, of the absolute value of the spherical harmonic components. This latter spectrum clearly shows the large scale component of the mode $\ell \leq 60$ and the internal layers contribution at $\ell>60$.

The width of these shear layers is obviously controlled by dissipative effects while the amplitude of the flow (or magnetic perturbation) is not. A preliminary investigation of the width of these layers seems to show a scaling with $\mathrm{Ha}^{-1 / 3}$; the fact that the amplitude of the flow is independent of the diffusion parameters is conspicuous from the spectral signature of the layers which is clear in the spherical harmonic decomposition (see Fig. 10).

One consequence of this situation is that such layers may strongly damp some modes and therefore insidiously participate to the selection of visible modes. We can indeed conjecture that such modes have a damping rate scaling like:

$\tau=\left(E+E_{\mathrm{m}}\right)\left(\tau_{\mathrm{inv}}+A / E_{\mathrm{m}}^{\alpha}\right)$

where $\alpha \sim 1 / 3$ and $A \sim 10^{-3}$ from numerical investigations. The second term $A / E_{\mathrm{m}}^{\alpha}$ is an evaluation of the contribution of the internal layers. The rather small value of the $A$ coefficient shows that these layers do not influence the damping rates at high diffusivities but when these are reaching stellar values 
$E_{\mathrm{m}} \sim 10^{-8}$, their role may be important. Since their dynamic is quite involved, their study is left to future investigations.

\section{Discussion}

In this paper, we have considered the case of pure shear Alfvén waves propagating in an incompressible viscous conducting fluid bathed by a dipolar magnetic field. We have shown that the axisymmetric poloidal eigenmodes of this system are trapped around the magnetic poles and that they are composed of an adiabatic part and internal shear layers associated with resonant field lines. The adiabatic part most likely results from the trapping of Alfvén waves in the region with most intense magnetic fields. The resonant frequency of this "trapping cavity" is also a resonant frequency for some specific field lines; these lines generate internal shear/magnetic layers which may give an important contribution to the damping rates and therefore to the excitability of the modes.

Most calculations have been done using a thick spherical shell which is appropriate for a planetary core like the Earth's. However, the case of a thin layer shares the same properties as illustrated by Fig. 9. In this case the modes still stay on the polar caps but their horizontal extension is much larger than the vertical one. Also, the frequency increases like the inverse of the thickness of the layer as shown by Eq. (32).

Back to the question of the oscillations of roAp stars which motivated this work, we see that the dynamics of a magnetic layer is fairly more complex than previously thought and in our system only Alfvén modes are possible! Despite its simplicity our model is also interesting as it shows the role of the magnetic field in the localization of the oscillations around the magnetic pole (most of the amplitude is within $12^{\circ}$ around the magnetic pole). The localization also appears in the spherical harmonic decomposition of the modes which peaks at rather high orders $(\ell \sim 20)$. Such a feature of the mode, if it persist in more realistic models, is not incompatible with observations. Indeed, observations are usually interpreted with the oblique pulsator model where it is assumed that intensity fluctuations result from the excitation of low-degree high order acoustic modes. In many cases low degree means $\ell=1$ (e.g. Kurtz 1990). However, this interpretation is not unique as underlined by previous authors (e.g. Kurtz 1992). Another possibility is that the oscillation is localized on the polar caps and because of limb-darkening effects it is modulated by rotation in the same way as a $\ell=1$ mode.

Quite clearly more work is needed to grasp the full complexity of these systems. Here the exploration has been restricted to axisymmetric poloidal modes, but the knowledge of the complete spectrum calls for an investigation of both purely toroidal axisymmetric modes and non-axisymmetric modes; these latter modes, which are numerically much more demanding, will be presented in subsequent work. Finally, it is clear that the hypothesis of incompressibility need to be relaxed, first for the allowance of a variable density background but also to fully appreciate the interaction of acoustic and magnetic modes.

Acknowledgements. We are pleased to acknowledge many fruitful discussions with Katia Ferrière, Boris Dintrans and Lorenzo
Valdettaro. The numerical calculations have been carried out on the NEC SX5 of the "Institut du Développement et des Ressources en Informatique Scientifique" (IDRIS) and on the CalMip machine of CICT in Toulouse which are both gratefully acknowledged.

\section{Appendix A: Spherical harmonics expansion of the MHD equations}

In this appendix, we expand each term of Eq. (10) on the spherical harmonics using Eq. (14) when $\boldsymbol{B}$ is a dipolar field. The $\boldsymbol{S}_{\ell}^{m}$ components are omitted because they can be deduced from the incompressibility and magnetic flux conservation conditions according to:

$$
\begin{array}{ll}
\boldsymbol{\nabla} \cdot \boldsymbol{v}=0 \quad & \Rightarrow \quad v_{m}^{\ell}=\frac{1}{\ell(\ell+1) r} \frac{\partial r^{2} u_{m}^{\ell}}{\partial r} \\
\boldsymbol{\nabla} \cdot \boldsymbol{b}=0 \quad \Rightarrow \quad b_{m}^{\ell}=\frac{1}{\ell(\ell+1) r} \frac{\partial r^{2} a_{m}^{\ell}}{\partial r}
\end{array}
$$

The following notations are used:

$$
\begin{aligned}
& B_{r}(r)=\frac{1}{r^{3}} \\
& B_{r}^{\prime}(r)=-\frac{3}{r^{4}} \\
& B_{\theta}(r)=\frac{1}{2 r^{3}} \\
& B_{\theta}^{\prime}(r)=-\frac{3}{2 r^{4}} \\
& A_{\ell-1}^{\ell}=\frac{1}{\ell^{2}} \sqrt{\frac{\ell^{2}-m^{2}}{(2 \ell-1)(2 \ell+1)}} \\
& A_{\ell+1}^{\ell}=\frac{1}{\ell^{2}} \sqrt{\frac{(\ell+1)^{2}-m^{2}}{(2 \ell+1)(2 \ell+3)}}
\end{aligned}
$$

$$
\begin{aligned}
\boldsymbol{\nabla} \times \boldsymbol{v}= & \frac{\ell(\ell+1)}{r} \quad w_{m}^{\ell} \boldsymbol{R}_{\ell}^{m} \\
& +\left\{\begin{array}{lr}
\frac{1}{r}\left(1-\frac{2}{\ell(\ell+1)}\right) & u_{m}^{\ell} \\
-\frac{4}{\ell(\ell+1)} & \frac{\partial u_{m}^{\ell}}{\partial r} \\
-\frac{r}{\ell(\ell+1)} & \frac{\partial^{2} u_{m}^{\ell}}{\partial r^{2}}
\end{array}\right\} \boldsymbol{T}_{\ell}^{m}
\end{aligned}
$$

$$
\boldsymbol{\nabla} \times \Delta \boldsymbol{v}=\frac{\ell(\ell+1)}{r}\left\{\begin{array}{lr}
-\frac{\ell(\ell+1)}{r^{2}} & w_{m}^{\ell} \\
+\frac{2}{r} & \frac{\partial w_{m}^{\ell}}{\partial r_{\ell}} \\
+1 & \frac{\partial^{2} w_{m}^{\ell}}{\partial r^{2}}
\end{array}\right\} \boldsymbol{R}_{\ell}^{m}
$$

$$
+\left\{\begin{array}{lr}
\frac{1}{r^{3}}(2-\ell(\ell+1)) & u_{m}^{\ell} \\
+\frac{4}{r^{2}} & \frac{\partial u_{m}^{\ell}}{\partial r} \\
+\frac{1}{r}\left(2-\frac{12}{\ell(\ell+1)}\right) & \frac{\partial^{2} u_{m}^{\ell}}{\partial r^{2}} \\
-\frac{8}{\ell(\ell+1)} & \frac{\partial^{3} u_{m}^{\ell}}{\partial r^{3}} \\
-\frac{r}{\ell(\ell+1)} & \frac{\partial^{4} u_{m}^{\ell}}{\partial r^{4}}
\end{array}\right\} \boldsymbol{T}_{\ell}^{m}
$$




$$
\begin{aligned}
& \boldsymbol{\nabla} \times((\boldsymbol{\nabla} \times \boldsymbol{b}) \times \boldsymbol{B})=\frac{\ell(\ell+1)}{r}\left\{\begin{array}{ll}
\left((\ell+1)(\ell+2) B_{r}(r)-(\ell+2)(\ell+1)^{2} B_{\theta}(r)\right) \frac{A_{\ell+1}^{\ell}}{r} & c_{m}^{\ell+1} \\
+(\ell+1)(\ell+2) A_{\ell+1}^{\ell} B_{r}(r) & \frac{\partial c_{m}^{\ell+1}}{\partial r} \\
+\left(\ell(\ell-1) B_{r}(r)+\ell^{2}(\ell-1) B_{\theta}(r)\right) \frac{A_{\ell-1}^{\ell}}{r} & c_{m}^{\ell-1} \\
+\ell(\ell-1) A_{\ell-1}^{\ell} B_{r}(r) & \frac{\partial c_{m}^{\ell-1}}{\partial r} \\
+\left(\frac{i m}{\ell(\ell+1)} \frac{B_{r}(r)}{r}-2 \frac{i m}{\ell^{2}(\ell+1)^{2}} \frac{B_{r}(r)}{r}\right) & \frac{a_{m}^{\ell}}{2 a_{m}^{\ell}} \\
-4 \frac{i m}{\ell^{2}(\ell+1)^{2}} B_{r}(r) & \frac{\partial^{2} a_{m}^{\ell}}{\partial r^{2}}
\end{array}\right\} \boldsymbol{R}_{\ell}^{m} \\
& +\left\{\begin{array}{lr}
\left(\frac{\ell B_{\theta}(r)}{r^{2}}+\frac{B_{r}^{\prime}(r)}{r}\right)(\ell+1)(\ell-2) A_{\ell-1}^{\ell} & a_{m}^{\ell-1} \\
+\left((\ell+2)(\ell-3) \frac{B_{r}(r)}{r}-4 B_{r}^{\prime}(r)-\frac{4 \ell B_{\theta}(r)}{r}\right) A_{\ell-1}^{\ell} & \frac{\partial a_{m}^{\ell-1}}{\partial r} \\
-\left(\ell B_{\theta}(r)+B_{r}^{\prime}(r) r+6 B_{r}(r)\right) A_{\ell-1}^{\ell} & \frac{\partial^{2} a_{m}^{\ell-1}}{\partial r^{2}} \\
-B_{r}(r) r A_{\ell-1}^{\ell} & \frac{\partial^{3} a_{m}^{\ell-1}}{\partial r^{3}} \\
-\left(\frac{(\ell+1) B_{\theta}(r)}{r^{2}}-\frac{B_{r}^{\prime}(r)}{r}\right) \ell(\ell+3) A_{\ell+1}^{\ell} & a_{m}^{\ell+1} \\
+\left((\ell+4)(\ell-1) \frac{B_{r}(r)}{r}-4 B_{r}^{\prime}(r)+\frac{4(\ell+1) B_{\theta}(r)}{r}\right) A_{\ell+1}^{\ell} & \frac{\partial a_{m}^{\ell+1}}{\partial r} \\
+\left((\ell+1) B_{\theta}(r)-B_{r}^{\prime}(r) r-6 B_{r}(r)\right) A_{\ell+1}^{\ell} & \frac{\partial^{2} a_{m}^{\ell+1}}{\partial r^{2}} \\
-B_{r}(r) r A_{\ell+1}^{\ell} & \frac{\partial^{3} a_{m}^{\ell+1}}{\partial r^{3}} \\
+\left(\frac{i m B_{\theta}^{\prime}(r)}{r}-\frac{i m B_{r}^{\prime}(r)}{\ell(\ell+1) r}-\frac{i m B_{\theta}(r)}{r^{2}}\right) & c_{m}^{\ell} \\
-\left(\frac{2 i m B_{r}(r)}{\ell(\ell+1) r}+\frac{i m B_{r}^{\prime}(r)}{\ell(\ell+1)}\right) & \frac{\partial c_{m}^{\ell}}{\partial r} \\
-\frac{i m B_{r}(r)}{\ell(\ell+1)} & \frac{\partial^{2} c_{m}^{\ell}}{\partial r^{2}}
\end{array}\right\} \\
& \boldsymbol{\nabla} \times(\boldsymbol{v} \times \boldsymbol{B})=\frac{\ell(\ell+1)}{r}\left\{\begin{array}{lr}
\left(2 B_{r}(r)+\ell B_{\theta}(r)\right) A_{\ell-1}^{\ell} & \frac{u_{m}^{\ell-1}}{+B_{r}(r) r A_{\ell-1}^{\ell}} \\
+\left(2 B_{r}(r)-(\ell+1) B_{\theta}(r)\right) A_{\ell+1}^{\ell} & \frac{u_{m}^{\ell+1}}{\ell r} \\
+B_{r}(r) r A_{\ell+1}^{\ell} & \frac{\partial u_{m}^{\ell+1}}{\partial r} \\
+\frac{i m B_{r}(r)}{\ell(\ell+1)} & w_{m}^{\ell}
\end{array}\right\} \boldsymbol{R}_{\ell}^{m} \\
& +\left\{\begin{array}{lr}
\left(\frac{\ell^{2}(\ell-1) B_{\theta}(r)}{r}+\frac{\ell(\ell-1) B_{r}(r)}{r}+\ell(\ell-1) B_{r}^{\prime}(r)\right) A_{\ell-1}^{\ell} & w_{m}^{\ell-1} \\
+\ell(\ell-1) B_{r}(r) A_{\ell-1}^{\ell} & \frac{\partial w_{m}^{\ell-1}}{\partial r} \\
+\left(\frac{(\ell+1)(\ell+2) B_{r}(r)}{r}+(\ell+1)(\ell+2) B_{r}^{\prime}(r)-\frac{(\ell+1)^{2}(\ell+2) B_{\theta}(r)}{r}\right) A_{\ell+1}^{\ell} & w_{m}^{\ell+1} \\
+(\ell+1)(\ell+2) B_{r}(r) A_{\ell+1}^{\ell} & \frac{\partial w_{m}^{\ell+1}}{\partial r} \\
+\left(\frac{i m B_{\theta}^{\prime}(r)}{\ell(\ell+1)}-\frac{i m B_{\theta}(r)}{\ell(\ell+1) r}-\frac{2 i m B_{r}^{\prime}(r)}{\ell^{2}(\ell+1)^{2}}-\frac{2 i m B_{r}(r)}{\ell^{2}(\ell+1)^{2} r}\right) & u_{m}^{\ell} \\
-\left(\frac{4 i m B_{r}(r)}{\ell^{2}(\ell+1)^{2}}+\frac{i m B_{r}^{\prime}(r) r}{\ell^{2}(\ell+1)^{2}}\right) & \frac{\partial u_{m}^{\ell}}{\partial r} \\
-\frac{i m B_{r}(r) r}{\ell^{2}(\ell+1)^{2}} & \frac{\partial^{2} u_{m}^{\ell}}{\partial r^{2}}
\end{array}\right\} \boldsymbol{T}_{\ell}^{m}
\end{aligned}
$$




$$
\begin{aligned}
\Delta \boldsymbol{b}= & \left\{\begin{array}{ll}
\frac{1}{r^{2}}(2-\ell(\ell+1)) & a_{m}^{\ell} \\
+\frac{4}{r} & \frac{\partial a_{m}^{\ell}}{\partial r} \\
+1 & \frac{\partial^{2} a_{m}^{\ell}}{\partial r^{2}}
\end{array}\right\} \boldsymbol{R}_{\ell}^{m} \\
& +\left\{\begin{array}{ll}
-\frac{\ell(\ell+1)}{r^{2}} & c_{m}^{\ell} \\
+\frac{2}{r} & \frac{\partial c_{m}^{\ell}}{\partial r} \\
+1 & \frac{\partial^{2} c_{m}^{\ell}}{\partial r^{2}}
\end{array}\right\} \boldsymbol{T}_{\ell}^{m} .
\end{aligned}
$$

\section{Appendix B: A toy model for understanding the trapping of Alfvén waves}

We derive a simple model to study the trapping of Alfvén waves when a non uniform magnetic field $\boldsymbol{B}=B(x) \boldsymbol{e}_{z}$ (cartesian geometry) is applied. The plasma layer has an infinite horizontal extent and is assumed to be inviscid and perfectly conducting. The magnetic field is maintained by a permanent electric current $\boldsymbol{J}=-\partial_{x} B \boldsymbol{e}_{y}$. Taking the curl of the momentum equation and using the fact that the problem does not depend on the $y$ coordinate, we obtain

$i \omega\left(\frac{\partial v_{x}}{\partial z}-\frac{\partial v_{z}}{\partial x}\right)=B \frac{\partial j_{y}}{\partial z}+b_{x} \frac{\partial J_{y}}{\partial x}$

where small case letters represent small perturbations. The induction equation shows that

$i \omega b_{x}=B \frac{\partial v_{x}}{\partial z}$

and

$i \omega j_{y}=B \frac{\partial^{2} v_{x}}{\partial z^{2}}+2 B^{\prime} \frac{\partial v_{x}}{\partial x}+B^{\prime \prime} v_{x}+B \frac{\partial^{2} v_{x}}{\partial x^{2}}$.

Next we differentiate Eq. (B.1) with respect to $x$ and make use of the incompressibility condition. Then, combining with Eqs. (B.2) and (B.3), we finally find an equation involving the horizontal velocity perturbation $v_{x}$ only (primes stand for the $x$-derivatives of the background field).

$$
-\frac{\omega^{2}}{B^{2}} \Delta v_{x}=\frac{\partial^{2}}{\partial z^{2}}\left(\Delta v_{x}+\frac{2 B^{\prime}}{B} \frac{\partial v_{x}}{\partial x}\right) .
$$

As the non-uniformity of the magnetic field is in the horizontal direction, we can consider a plane-wave expansion in the $z$ direction, i.e. $\partial_{z} v_{x}=i k_{\|} v_{x}$. Using this in Eq. (B.4), a second order differential equation in $x$ can be obtained

$\frac{\partial^{2} v_{x}}{\partial x^{2}}+\frac{2 k_{\|}^{2} B^{\prime}}{B\left(k_{\|}^{2}-\frac{\omega^{2}}{B^{2}}\right)} \frac{\partial v_{x}}{\partial x}-k_{\|}^{2} v_{x}=0$

which is the same as Eq. (33).

\section{References}

Balmforth, N. J., Cunha, M. S., Dolez, N., Gough, D. O., \& Vauclair, S. 2001, MNRAS, 323, 362

Bender, C. M., \& Orzag, S. A. 1978, Advanced mathematical methods for scientists and engineers (Mc Graw-Hill)

Bigot, L., \& Dziembowski, W. A. 2002, A\&A, 391, 235

Bigot, L., Provost, J., Berthomieu, G., Dziembowski, W. A., \& Goode, P. R. 2000, A\&A, 356, 218

Biront, D., Goosens, M., Cousens, A., \& Mestel, L. 1982, MNRAS, 201, 619

Campbell, C. G., \& Papaloizou, J. C. B. 1986, MNRAS, 220, 577

Chandrasekhar, S. 1961, Hydrodynamic and hydromagnetic stability (Dover)

Cox, J. P. 1984, ApJ, 280, 220

Cummings, W. D., \& O'Sullivan, R. J. 1969, J. Geophys. Res., 74, 778

Dziembowski, W. A., \& Goode, P. R. 1996, ApJ, 458, 338

Friedlander, S. 1987, Geophys. Astrophys. Fluid Dyn., 39, 315

Friedlander, S. 1989, Geophys. Astrophys. Fluid Dyn., 48, 53

Kurtz, D. 1992, MNRAS, 259, 701

Kurtz, D. W. 1990, ARA\&A, 28, 607

Malkus, W. V. R. 1967, J. Fluid Mech., 28, 793

Mc Tavish, J. P. 2000, Am. J. Phys., 68, 577

Mendell, G. 1998, MNRAS, 296, 903

Potherat, A., Sommeria, J., \& Moreau, R. 2002, Phys. Fluids, 14, 403

Radoski, H. R. 1967, J. Geophys. Res., 72, 418

Rieutord, M. 1987, Geophys. Astrophys. Fluid Dyn., 39, 163

Rieutord, M. 1991, Geophys. Astrophys. Fluid Dyn., 59, 182

Rieutord, M., Georgeot, B., \& Valdettaro, L. 2001, J. Fluid Mech., 435,103

Rieutord, M., \& Valdettaro, L. 1997, J. Fluid Mech., 341, 77

Roberts, P. H., \& Soward, A. M. 1983, MNRAS, 205, 1171

Shibahashi, H. 1983, ApJ, 275, L5 